\title{
Southern triplets of galaxies
}

\author{
V. E. Karachentseva \\ Astronomical Observatory of Kiev University, Ukraine
}

\section{D. Karachentsev}

Special Astrophysical Observatory, Russia

\begin{abstract}
Using the ESO/SERC and POSS-I sky surveys we selected 76 isolated triple systems of galaxies with Dec. $<-3^{\circ}$. For each triplet the equatorial coordinates, type of configuration, angular diameters, apparent angular separation of the components, morphological types, total magnitudes and some other characteristics are presented. 33 of 76 triplets have the measured radial velocities for all the components. The median values of basic dynamic parameters: a radial velocity dispersion, mean harmonic separation, an absolute magnitude of galaxies, mass-toluminosity ratio are very close to those obtained earlier for 83 northern isolated triple systems from the list of Karachentseva et al. (1979).
\end{abstract}

\section{Introduction}

The first published list of isolated galaxy triplets (Karachentseva et al., 1979) contains 83 triple systems of galaxies with apparent magnitudes $m_{p} \leq 15.7$ and declinations $\delta>-3^{\circ}$. The members of triple systems were selected on the basis of the Palomar Sky Atlas and Zwicky et al. (1968) Catalogue. Three galaxies were considered to form an isolated triplet if their "significant" neighbors were at least three times as far away from them as the components of the triplet were from one another. The significant neighbors were neighboring galaxies whose angular diameters differed by a factor $(1 / 2-2)$ of the diameter of a triplet member.

Triple system membership of galaxies was determined disregarding to their radial velocities. Therefore a system may be isolated only in projection on the sky but, generally, not in three-dimensional space. The radial velocities of all members of triplets have been measured mainly with the 6-m telescope of SAO. That allowed to compile the catalogue of northern triple systems (Karachentseva et al., 1988) and determine their dynamical parameters (Karachentsev et al., 1989).

Over the last years this complete and homogeneous sample has been used as the observational basis to study the formation and evolution of triple galaxy systems by computer simulation methods. The results obtained by teams from Finland, Russia, and USA have been reviewed in Karachentsev et al. (2000). Due to the obvious importance of the specific kinds of small galaxy groups for 
the study of the dynamical evolution of groups of galaxies, we have performed a search for isolated triple systems in the southern hemisphere with almost the same selection criterion which have been used for the northern triplets (hereafter NTs).

\section{Description of the sample of southern isolated triplets}

For selection of isolated southern triplets in the declination range $\{-20,-90\}^{\circ}$ we used the ESO/SERC Sky Survey as well as the "ESO/Uppsala Survey of the ESO(B) Atlas" by Lauberts (1982) with a limiting angular diameter of galaxies $\simeq 1^{\prime}$. In the declination range $\{-3,-20\}^{\circ}$ we used the POSS-I Sky Survey, and the Morphological Catalogue of Galaxies (hereafter MCG) by VorontsovVelyamonov and Arkhipova $(1963,1968)$ having an apparent magnitude limit $\simeq 15$ mag. The fields with Galactic latitude $b<20^{\circ}$ were not considered.

The above described criteria of local isolation have been applied to all galaxies from Lauberts' Catalogue as well to those galaxies from MCG whose angular diameters exceed $0.85^{\prime}$. The difference in the limiting galaxy diameters is caused by the deeper photometric limit of the ESO/SERC survey with respect to POSSI. In total we found 76 isolated triple systems containing about 1 percent of the number of galaxies, i.e. almost the same percentge as for the northern sky. The resulting list of southern triplets (Karachentseva \& Karachentsev, 1999) is organized in columns as follows (see Table 2):

1 The triplet number (TS) and letter designation of its components A, B, C arranged by increasing right ascension.

2 Satisfaction of the isolation criterion (Is) for each component with + as yes and - as no. About $2 / 3$ of the triple systems are fully isolated $(+++)$. For NTs this share is $64 \%$.

3 Right ascension and declination at epoch 1950.0. For the MCG galaxies and for galaxies absent in the ESO/Uppsala Catalogue, coordinates were measured on the DigSS.

4, 5 "Blue" angular diameter $a$ (major axis) and $b$ (minor axis) in arcmin referring to the maximum extent of features which we described as belonging to the object. The diameters measured on the ESO/SERC films have been translated into the POSS-I system according to the relations from Kudrya et al. (1997): $a_{O}=0.8078 \cdot a_{J}$ and $b_{O}=0.7827 \cdot b_{J}$.

6 Apparent angular separations $x_{i, k}$ of the components in arcmin;

7 Configuration (C) of the triplet: D - double or hierarchical (one of the sides of the apparent triangle is $1 / 3$ or less than each of the two other sides); L - linear (one of the angles of the apparent triangle is more than $150^{\circ}$ ); $\mathrm{T}$ - triangle (all the three sides and angles are comparable). The distribution of triplets according to their configuration is the following: $34 \%(\mathrm{D}), 42 \%(\mathrm{~T})$, and $24 \%(\mathrm{~L})$. (For NTs these ratios are: $38 \%(\mathrm{D})$, $49 \%(\mathrm{~T})$, and $13 \%(\mathrm{~L}))$. Figs $1(\mathrm{a})$ and (b) show the different types of triple galaxy configurations. 
8 Morphological type (Ty) of the galaxies, one of 6 classes: $\mathrm{E}, \mathrm{S} 0, \mathrm{Sa}, \mathrm{Sb}$, $\mathrm{Sc}, \mathrm{Sm}+\mathrm{Pec}$ distributed (in percent) as 9:13:17:31:19:11. (For NTs they are $16: 12: 21: 28: 18: 5)$.

9 The galaxy number in other catalogues. Note that $12 \%$ of galaxies entering in our list were uncatalogued before.

10 Total apparent magnitude of the galaxy taken from the LEDA database.

11 Radial velocity $(v)$ in $\mathrm{km} \mathrm{s}^{-1}$, together with internal error, $\sigma_{v}$, of the radial velocity measurements also taken from the LEDA. About $60 \%$ of galaxies entering in the southern triplets have the measured radial velocities.

The whole sky distribution of the centres of 159 northern and southern triplets in equatorial coordinates is presented in Fig. 2. The distibution seems to be rather homogeneous with about the same surface number density for both hemispheres.

\section{Dynamical parameters of the triplets}

Among 76 southern triplets 33 have radial velocity measurements for all three components. For them we calculated the main dynamical characteristics using the same scheme as for northern triplets (Karachentsev et al., 1989, Karachentsev et al., 1999). Each triplet as a dynamical system is characterized by the following parameters:

- the average centroid radial velocity, $\langle v\rangle$, corrected for the Solar motion;

- the rms velocity of triple galaxies relative to their center, $s_{v}^{2}$;

- the mean harmonic separation in projection, $r_{H}$ in kpc with the Hubble constant $H=75 \mathrm{~km} \mathrm{~s}^{-1} \mathrm{Mpc}^{-1}$;

- the luminosity of galaxy in solar units, where the absolute magnitude of the galaxy is determined from the mean velocity and the apparent magnitude corrected for the galactic extinction (Schlegel et al., 1998) and internal absorption in the galaxy;

- the dimensionless crossing time of the triplets expressed in terms of the Hubble time $\left(H^{-1}\right)$;

- the virial mass-to-luminosity ratio for the triple system, $f$;

- the statistically unbiased estimate of the same ratio, $f^{c}$.

In Table 1 we present the median values of the main dynamical parameters for the southern triplets in comparison with the northern ones. We compare the data for the entire samples (for STs it is only $42 \%$ of the sample with known radial velocities) as well as for "physical" triplets with $s_{v}<300 \mathrm{~km} \mathrm{~s}^{-1}$. Both subsamples have nearly the same global parameters. However, detailed analysis of the data is only reasonable when radial velocities are determined for all components of the southern triple systems. 


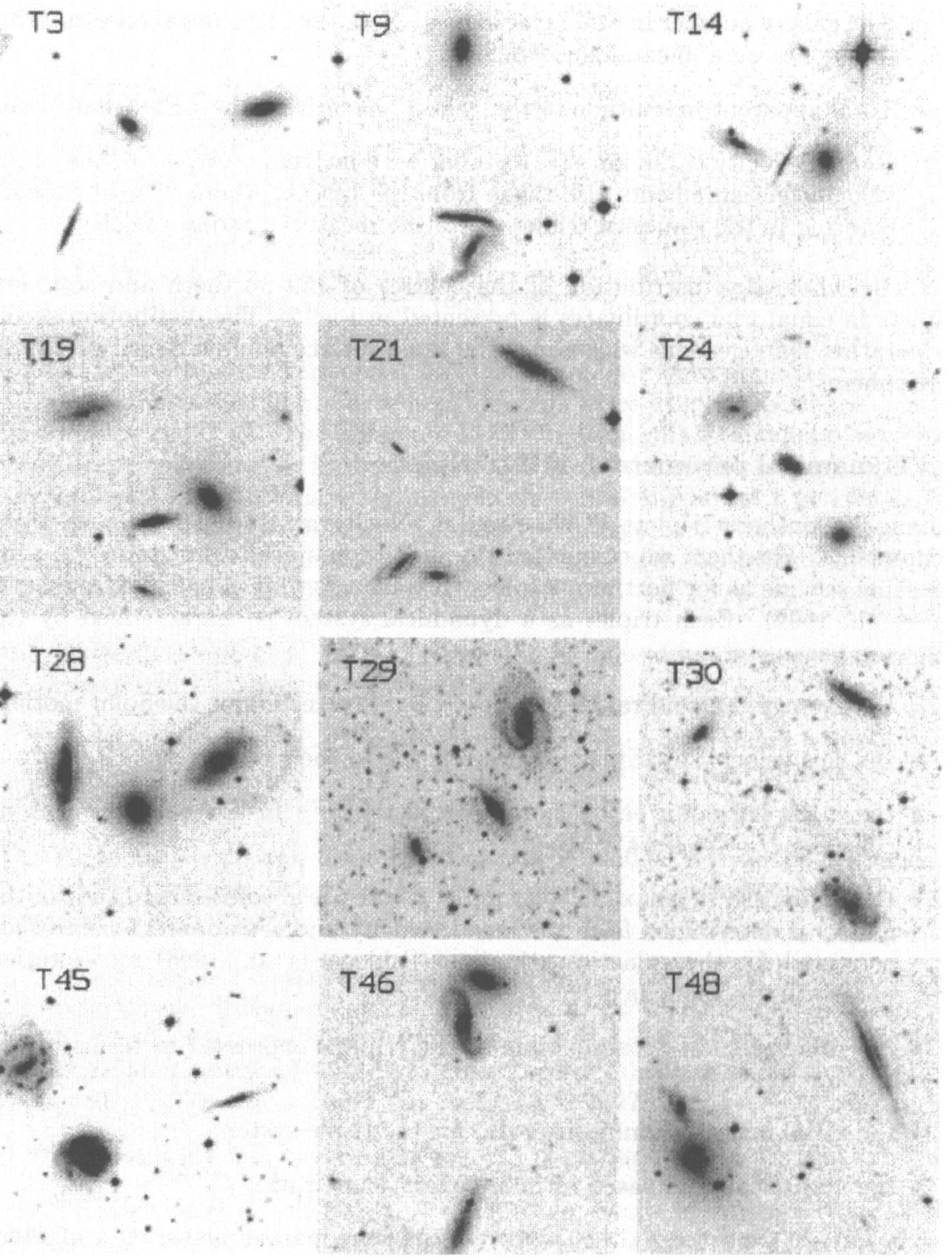

Figure 1(a). The images of the southern triplets from the digitized POSS-I. Each panel is 5 arcmin on a side. North is at the top, and east is to the left. 


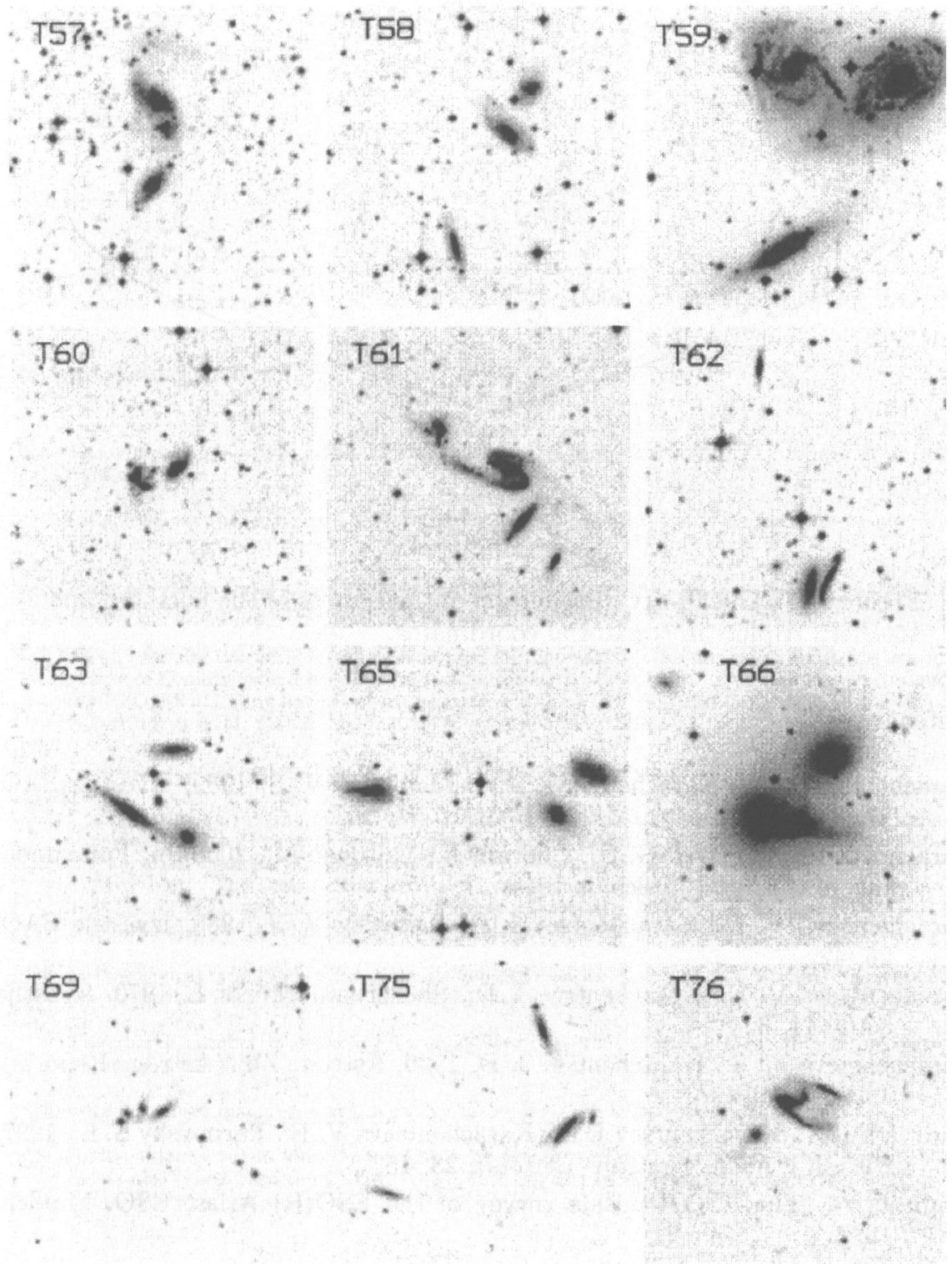

Figure 1(b). Further images of southern triplets from the digitized POSS-I. Image size and orientation as for Fig 1(a) 


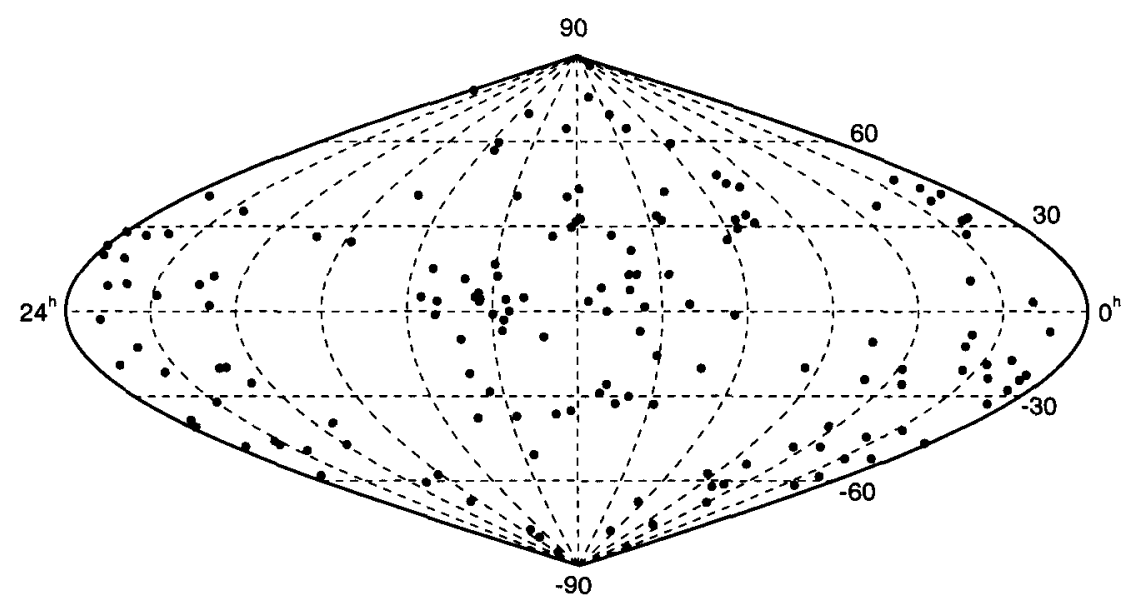

Figure 2. The all-sky distribution of the centres of the northern and southern triplets in equatorial coordinates.

\section{References}

Karachentsev I. D., Karachentseva V. E., Lebedev V. S. 1989, Izvestija SAO, 27,67 .

Karachentsev I. D., Byrd G. G., Chernin A., Valtonen M., 2000, in: Fundamentals of Cosmic Physics, in press.

Karachentseva V. E., Karachentsev I. D., Lebedev V. S. 1988, Izvestija SAO, 26,42 .

Karachentseva V. E., Karachentsev I. D., Shcherbanivskii A. L. 1979, Izvestija $\mathrm{SAO}, 11,3$.

Karachentseva V. E., Karachentsev I. D. 1999, Astron. Zh. (Astron. Reports), 1999, submitted

Kudrya Yu. N., Karachentsev I. D., Karachentseva V. E., Parnovsky S. L., 1997, Pis'ma v A.Zh. (Astron. Letters), 23, 15.

Lauberts A., The ESO/Uppsala survey of the ESO(B) Atlas, ESO, Munich, 1982.

Schlegel D. J., Finkbeier D. P., Davis M., 1998, AJ,500, 525.

Vorontsov-Velyaminov B. A., Arkhipova V. P. Morphological Catalogue of Galaxies, 1963, 1968, pp.III-IV, Moscow Univ., Moscow.

Zwicky F., Herzog E., Wild P., Karpowicz M., Kowal C., 1968, Catalog of Galaxies and of Clusters of Galaxies: VV. I-VI, Cal. Inst. Tech., Pasadena. 
Table 1. Medians of some parameters of triplets

\begin{tabular}{|c|cc|cc|}
\hline Parameter & $\begin{array}{c}\text { Present paper } \\
\text { Southern } \\
\mathrm{N}=33\end{array}$ & $\begin{array}{c}\text { All } \\
\text { Northern } \\
\mathrm{N}=83\end{array}$ & \multicolumn{2}{|c|}{$s_{v}<300 \mathrm{~km} \mathrm{~s}^{-1}$} \\
Southern & $\mathrm{N}=24$ & $\begin{array}{c}\text { Northern } \\
\mathrm{N}=53\end{array}$ \\
\hline$s_{v}\left(\mathrm{~km} \mathrm{~s}^{-1}\right)$ & 166 & 133 & 119 & 100 \\
$r_{H}(\mathrm{kpc})$ & 72 & 63 & 60 & 55 \\
$L\left(L_{\odot}\right)$ & $7.1 \times 10^{10}$ & $7.4 \times 10^{10}$ & $6.3 \times 10^{10}$ & $7.6 \times 10^{10}$ \\
$\tau\left(H^{-1}\right)$ & 0.08 & 0.04 & 0.08 & 0.07 \\
$f\left(f_{\odot}\right)$ & 102 & 67 & 64 & 31 \\
$f^{c}\left(f_{\odot}\right)$ & 99 & 58 & 44 & 27 \\
\hline
\end{tabular}

\section{Notes to Table 2: Southern Isolated Triplets of Galaxies}

1 The triplet number (TS) and letter designation of its components A, B, C arranged by increasing right ascension.

2 Satisfaction of the isolation criterion (Is) for each component with + as yes and - as no. About $2 / 3$ of the triple systems are fully isolated $(t++)$. For NTs this share is $64 \%$.

3 Right ascension and declination at epoch 1950.0. For the MCG galaxies and for galaxies absent in the ESO/Uppsala Catalogue, coordinates were measured on the DigSS.

4, 5 "Blue" angular diameter $a$ (major axis) and $b$ (minor axis) in arcmin referring to the maximum extent of features which we described as belonging to the object. The diameters measured on the ESO/SERC films have been translated into the POSS-I system according to the relations from Kudrya et al. (1997): $a_{O}=0.8078 \cdot a_{J}$ and $b_{O}=0.7827 \cdot b_{J}$.

6 Apparent angular separations $x_{i, k}$ of the components in arcmin;

7 Configuration (C) of the triplet: D - double or hierarchical (one of the sides of the apparent triangle is $1 / 3$ or less than each of the two other sides); $\mathrm{L}$ - linear (one of the angles of the apparent triangle is more than $150^{\circ}$ ); $\mathrm{T}$ - triangle (all the three sides and angles are comparable). The distribution of triplets according to their configuration is the following: $34 \%(\mathrm{D}), 42 \%(\mathrm{~T})$, and $24 \%(\mathrm{~L})$. (For NTs thes e ratios are: $38 \%(\mathrm{D}), 49 \%(\mathrm{~T})$, and $13 \%(\mathrm{~L})$ ). Figs 1 (a) and (b) show the different types of triple galaxy configurations.

8 Morphological type (Ty) of the galaxies, one of 6 classes: $\mathrm{E}, \mathrm{S} 0, \mathrm{Sa}, \mathrm{Sb}, \mathrm{Sc}, \mathrm{Sm}+\mathrm{Pec}$ distributed (in percent) as 9:13:17:31:19:11. (For NTs they are 16:12:21:28:18:5).

9 The galaxy number in other catalogues. Note that $12 \%$ of galaxies entering in our list were uncatalogued before.

10 Total apparent magnitude of the galaxy taken from the LEDA database.

11 Radial velocity $(v)$ in $\mathrm{km} \mathrm{s}^{-1}$, together with internal error, $\sigma_{v}$, of the radial velocity measurements also taken from the LEDA. About $60 \%$ of galaxies entering in the southern triplets have the measured radial velocities. 
Table 2. List of southern isolated triplets of galaxies

\begin{tabular}{|c|c|c|c|c|c|c|c|c|c|c|}
\hline $\mathrm{TS}$ & Is. & R.A.(1950) Dec. & $a$ & $b$ & $X_{i j}$ & C. & Type & Alias. & $\overline{B_{t}}$ & $v \pm \sigma$ \\
\hline 1 & 2 & 3 & 4 & 5 & 6 & 7 & 8 & 9 & 10 & 11 \\
\hline $1 \mathrm{~A}$ & + & $\begin{array}{llllll}00 & 07 & 49 & -46 & 41 & 50\end{array}$ & 2.17 & 0.54 & 4.47 & \multirow[t]{3}{*}{$\mathrm{D}$} & $\mathrm{SBb}$ & 241-21 & 14.26 & \multirow[t]{3}{*}{$6071 \pm 61$} \\
\hline $1 \mathrm{~B}$ & - & $00 \quad 0754-46 \quad 46 \quad 14$ & 1.08 & 0.45 & 4.71 & & SBbc & $241-22$ & 14.17 & \\
\hline $1 \mathrm{C}$ & + & 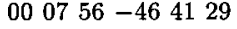 & 0.59 & 0.59 & 1.34 & & $\mathrm{Sc}$ & & & \\
\hline $2 \mathrm{~A}$ & + & $\begin{array}{llllll}0 & 33 & 40 & -32 & 52 & 48\end{array}$ & 0.90 & 0.45 & 1.94 & \multirow[t]{3}{*}{$\mathrm{D}$} & Sa & $350-37$ & 14.49 & \\
\hline $2 \mathrm{~B}$ & - & $\begin{array}{llllll}00 & 33 & 44 & -32 & 51 & 03\end{array}$ & 0.68 & 0.39 & 11.27 & & $\mathrm{Sa}$ & & & \\
\hline $2 \mathrm{C}$ & + & $\begin{array}{lllllll}00 & 34 & 36 & -32 & 48 & 17\end{array}$ & 1.18 & 0.45 & 12.58 & & So-a & $350-39$ & 15.83 & $14803 \pm 60$ \\
\hline $3 \mathrm{~A}$ & + & $\begin{array}{llllll}00 & 33 & 59 & -28 & 03 & 37\end{array}$ & 0.99 & 0.44 & 2.20 & \multirow[t]{3}{*}{$\mathrm{T}$} & S0-a & $410-24$ & 14.57 & \multirow{3}{*}{$\begin{array}{r}10431 \pm 52 \\
6910 \pm 52\end{array}$} \\
\hline 3B & + & $\begin{array}{llllll}0 & 34 & 09 & -28 & 03 & 49\end{array}$ & 0.99 & 0.57 & 2.01 & & E-So & $410-25$ & 15.09 & \\
\hline $3 \mathrm{C}$ & + & $\begin{array}{llllll}00 & 34 & 14 & -28 & 05 & 32\end{array}$ & 0.86 & 0.09 & 3.81 & & $\mathrm{Sc}$ & $410-26$ & 16.29 & \\
\hline $4 \mathrm{~A}$ & + & $\begin{array}{llllll}00 & 34 & 10 & -22 & 52 & 06\end{array}$ & 1.08 & 0.22 & 8.07 & \multirow[t]{3}{*}{$\mathbf{L}$} & S0-a & 474- 4 & 14.92 & $3890 \pm 60$ \\
\hline 4B & + & $003445-225136$ & 2.26 & 0.27 & 5.31 & & SBbc & 474- 5 & 14.08 & $2965 \pm 62$ \\
\hline $4 \mathrm{C}$ & + & $\begin{array}{lllll}0035 & 06 & -22 & 49 & 24\end{array}$ & 2.26 & 0.53 & 13.17 & & Sab & 474- 6 & 14.16 & $3858 \pm 54$ \\
\hline $5 \mathrm{~A}$ & + & $003604-243654$ & 1.18 & 0.44 & 3.93 & \multirow[t]{3}{*}{$\mathrm{T}$} & SBb & 474- 8 & 14.98 & $3764 \pm 9$ \\
\hline $5 \mathrm{~B}$ & + & 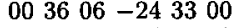 & 1.72 & 1.67 & 4.44 & & $\mathrm{SBc}$ & 474- 9 & 13.62 & $3681 \pm 39$ \\
\hline $5 \mathrm{C}$ & - & $003620-242952$ & 0.54 & 0.44 & 7.89 & & Sdm & & & \\
\hline $6 \mathrm{~A}$ & + & $004423-52 \quad 2302$ & 0.82 & 0.17 & 4.74 & \multirow[t]{3}{*}{$\mathrm{D}$} & $\mathrm{Sb}$ & & & \\
\hline $6 \mathrm{~B}$ & + & $004444-521928$ & 0.82 & 0.48 & 0.75 & & $\mathrm{Sa}\}$ & $194-39\}$ & $14.99\}$ & $8185 \pm 118$ \\
\hline $6 \mathrm{C}$ & + & $0044 \quad 49-521924$ & 0.90 & 0.10 & 5.35 & & Scd & & & \\
\hline $7 \mathrm{~A}$ & + & 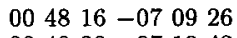 & 2.35 & 0.56 & 10.90 & \multirow[t]{3}{*}{ D } & S0 & $-1-3-19$ & 13.87 & $4753 \pm 60$ \\
\hline $7 \mathrm{~B}$ & + & 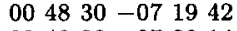 & 0.95 & 0.67 & 0.70 & & $\mathbf{E}$ & $-1-3-21$ & 12.71 & $1750 \pm 11$ \\
\hline $7 \mathrm{C}$ & + & 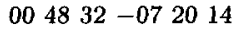 & 1.23 & 1.01 & 11.50 & & $\mathrm{SBC}$ & $-1-3-22$ & 12.63 & $1744 \pm 8$ \\
\hline $8 \mathrm{~A}$ & + & $\begin{array}{llllll}01 & 10 & 13 & -58 & 30 & 42\end{array}$ & 1.81 & 0.96 & 3.11 & \multirow[t]{3}{*}{$\mathrm{T}$} & SBab & $113-23$ & 12.77 & $4849 \pm 103$ \\
\hline $8 \mathrm{~B}$ & + & $\begin{array}{llllll}01 & 10 & 29 & -58 & 28 & 24\end{array}$ & 1.36 & 0.52 & 5.05 & & SO-a & $113-24$ & 15.66 & $4828 \pm 8$ \\
\hline $8 \mathrm{C}$ & + & 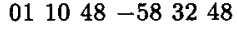 & 0.82 & 0.52 & 5.03 & & Sbc & $113-25$ & 13.67 & $5015 \pm 93$ \\
\hline $9 \mathrm{~A}$ & + & $01 \quad 1809-173930$ & 1.68 & 0.67 & 0.60 & \multirow[t]{3}{*}{ D } & $\mathrm{Sb}$ & $-3-4-53$ & 14.51 & $5961 \pm 48$ \\
\hline 9B & + & 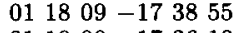 & 1.12 & 0.22 & 2.75 & & $\mathrm{SBb}$ & $-3-4-52$ & 14.67 & $5927 \pm 60$ \\
\hline $9 \mathrm{C}$ & + & 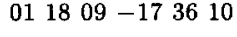 & 1.23 & 0.67 & 3.35 & & $\mathbf{E}$ & $-3-4-51$ & 14.45 & $9296 \pm 68$ \\
\hline $10 \mathrm{~A}$ & + & $\begin{array}{lllll}01 & 18 & 45 & -613536\end{array}$ & 1.08 & 0.79 & 6.71 & \multirow[t]{3}{*}{$\mathbf{D}$} & SBab & $113-40$ & 15.11 & \multirow{3}{*}{$8622 \pm 42$} \\
\hline $10 \mathrm{~B}$ & + & $\begin{array}{llllll}01 & 19 & 37 & -61 & 38 & 12\end{array}$ & 1.36 & 0.79 & 1.32 & & $\mathrm{SBb}$ & $113-41$ & 15.04 & \\
\hline $10 \mathrm{C}$ & + & $011939-613654$ & 0.82 & 0.61 & 6.55 & & E-SO & $113-42$ & 14.72 & \\
\hline $11 \mathrm{~A}$ & + & $\begin{array}{llllll}01 & 28 & 24 & -23 & 50 & 42\end{array}$ & 0.99 & 0.57 & 6.80 & \multirow[t]{3}{*}{$\mathbf{L}$} & $\mathrm{SBc}$ & $476-17$ & 14.91 & \\
\hline $11 \mathrm{~B}$ & + & 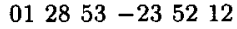 & 0.68 & 0.52 & 5.99 & & $\mathrm{Sa}$ & & & \\
\hline $11 \mathrm{C}$ & - & $012919-235123$ & 0.76 & 0.16 & 12.60 & & $\mathrm{Sbc}$ & & & \\
\hline $12 \mathrm{~A}$ & + & 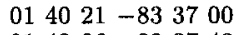 & 2.08 & 0.70 & 10.38 & \multirow[t]{3}{*}{$\mathrm{T}$} & $\mathrm{SBC}$ & 3- 3 & 14.56 & $4934 \pm 40$ \\
\hline $12 \mathrm{~B}$ & + & $\begin{array}{llllll}01 & 43 & 06 & -83 & 27 & 48\end{array}$ & 1.81 & 0.35 & 14.10 & & Sbc & $3-4$ & 14.85 & $4595 \pm 41$ \\
\hline $12 \mathrm{C}$ & + & $\begin{array}{llll}0148 & 13 & -833855\end{array}$ & 1.63 & 0.96 & 13.29 & & E-S0 & & & \\
\hline
\end{tabular}




\begin{tabular}{|c|c|c|c|c|c|c|c|c|c|c|}
\hline$\frac{T S}{1}$ & $\frac{1 s .}{2}$ & $\frac{\text { R.A. }(1950) \text { Dec. }}{3}$ & $\frac{a}{4}$ & $\frac{b}{5}$ & $\frac{X_{i j}}{6}$ & C. & $\frac{\text { Type }}{8}$ & $\frac{\text { Alias. }}{9}$ & $\begin{array}{l}B_{t} \\
10\end{array}$ & $\frac{v \pm \sigma}{11}$ \\
\hline $\begin{array}{l}13 \mathrm{~A} \\
13 \mathrm{~B} \\
13 \mathrm{C}\end{array}$ & $\begin{array}{l}+ \\
+ \\
+\end{array}$ & $\begin{array}{llllll}01 & 42 & 52 & -42 & 14 & 41 \\
01 & 43 & 03 & -42 & 07 & 48 \\
01 & 43 & 37 & -42 & 12 & 24\end{array}$ & $\begin{array}{l}0.90 \\
1.45 \\
0.90\end{array}$ & $\begin{array}{l}0.12 \\
0.88 \\
0.22\end{array}$ & $\begin{array}{l}7.19 \\
7.90 \\
8.64\end{array}$ & $\mathrm{~T}$ & $\begin{array}{l}\text { Sbc } \\
\text { Sb } \\
\text { Sbc }\end{array}$ & $\begin{array}{l}297-26 \\
297-27 \\
297-28\end{array}$ & $\begin{array}{l}16.08 \\
14.89 \\
16.51\end{array}$ & $6317 \pm 48$ \\
\hline $\begin{array}{l}14 \mathrm{~A} \\
14 \mathrm{~B} \\
14 \mathrm{C}\end{array}$ & $\begin{array}{l}+ \\
+ \\
+\end{array}$ & $\begin{array}{llllll}01 & 46 & 05 & -52 & 17 & 55 \\
01 & 46 & 10 & -52 & 17 & 57 \\
01 & 46 & 14 & -52 & 17 & 37\end{array}$ & $\begin{array}{l}0.72 \\
0.80 \\
0.99\end{array}$ & $\begin{array}{l}0.52 \\
0.07 \\
0.28\end{array}$ & $\begin{array}{l}0.67 \\
0.84 \\
1.46\end{array}$ & $\mathbf{L}$ & $\begin{array}{l}\text { SO-E } \\
\text { Sc\} } \\
\text { Sa }\end{array}$ & $197-1$ & 15.32 & $14708 \pm 60$ \\
\hline $\begin{array}{l}15 \mathrm{~A} \\
15 \mathrm{~B} \\
15 \mathrm{C}\end{array}$ & $\begin{array}{l}+ \\
+ \\
+\end{array}$ & $\begin{array}{llllll}01 & 50 & 06 & -19 & 01 & 09 \\
01 & 50 & 17 & -19 & 01 & 39 \\
01 & 50 & 52 & -19 & 11 & 00\end{array}$ & $\begin{array}{l}1.12 \\
1.01 \\
1.34\end{array}$ & $\begin{array}{l}1.01 \\
0.67 \\
0.25\end{array}$ & $\begin{array}{c}2.63 \\
12.48 \\
14.67\end{array}$ & $\mathrm{D}$ & $\begin{array}{l}\text { Sc } \\
\text { Sc } \\
\text { Sa }\end{array}$ & $\begin{array}{l}-3-5-26 \\
-3-5-27 \\
-3-5-28\end{array}$ & $\begin{array}{l}14.35 \\
14.47 \\
15.22\end{array}$ & $14580 \pm 60$ \\
\hline $\begin{array}{l}16 \mathrm{~A} \\
16 \mathrm{~B} \\
16 \mathrm{C}\end{array}$ & $\begin{array}{l}+ \\
+ \\
+\end{array}$ & $\begin{array}{llllll}02 & 19 & 34 & -21 & 03 & 07 \\
02 & 19 & 42 & -20 & 58 & 20 \\
02 & 20 & 43 & -20 & 56 & 24\end{array}$ & $\begin{array}{l}1.90 \\
1.13 \\
2.99\end{array}$ & $\begin{array}{l}1.58 \\
0.61 \\
0.61\end{array}$ & $\begin{array}{l}5.15 \\
14.37 \\
17.45\end{array}$ & D & $\begin{array}{l}\text { Irr } \\
\text { Irr } \\
\text { SBd }\end{array}$ & $\begin{array}{l}545-7 \\
545-8 \\
545-10\end{array}$ & $\begin{array}{l}13.14 \\
14.15 \\
13.19\end{array}$ & $\begin{array}{r}1563 \pm 11 \\
1580 \pm 6 \\
1726 \pm 11\end{array}$ \\
\hline $\begin{array}{l}17 \mathrm{~A} \\
17 \mathrm{~B} \\
17 \mathrm{C}\end{array}$ & $\begin{array}{l}+ \\
+\end{array}$ & $\begin{array}{llllll}02 & 29 & 07 & -44 & 38 & 48 \\
02 & 29 & 07 & -44 & 36 & 36 \\
02 & 29 & 45 & -44 & 44 & 42\end{array}$ & $\begin{array}{l}1.08 \\
0.54 \\
1.63\end{array}$ & $\begin{array}{l}0.48 \\
0.48 \\
1.14\end{array}$ & $\begin{array}{c}2.20 \\
10.55 \\
8.98\end{array}$ & D & $\begin{array}{l}\mathrm{SBc} \\
\mathrm{Sc} \\
\text { So }\end{array}$ & $246-23$ & 13.70 & $\begin{array}{r}5127 \pm 60 \\
4775 \pm 9\end{array}$ \\
\hline $\begin{array}{l}18 \mathrm{~A} \\
18 \mathrm{~B} \\
18 \mathrm{C}\end{array}$ & $\begin{array}{l}+ \\
+ \\
-\end{array}$ & $\begin{array}{llllll}02 & 37 & 02 & -08 & 20 & 54 \\
02 & 37 & 57 & -08 & 38 & 52 \\
02 & 38 & 37 & -08 & 28 & 10\end{array}$ & $\begin{array}{l}2.69 \\
5.60 \\
2.58\end{array}$ & $\begin{array}{l}0.90 \\
4.14 \\
2.02\end{array}$ & $\begin{array}{l}22.56 \\
14.57 \\
24.60\end{array}$ & $\mathrm{~T}$ & $\begin{array}{l}\text { Sc } \\
\text { SBc } \\
\text { E }\end{array}$ & $\begin{array}{l}-1-7-27 \\
-2-7-54 \\
-1-7-34\end{array}$ & $\begin{array}{l}12.88 \\
11.46 \\
11.36\end{array}$ & $\begin{array}{r}1241 \pm 7 \\
1373 \pm 8 \\
1507 \pm 40\end{array}$ \\
\hline $\begin{array}{l}19 \mathrm{~A} \\
19 \mathrm{~B} \\
19 \mathrm{C}\end{array}$ & $\begin{array}{l}+ \\
+ \\
+\end{array}$ & $\begin{array}{lllllll}02 & 40 & 14 & -12 & 38 & 01 \\
02 & 40 & 18 & -12 & 38 & 22 \\
02 & 40 & 22 & -12 & 36 & 31\end{array}$ & $\begin{array}{l}1.12 \\
1.12 \\
1.34\end{array}$ & $\begin{array}{l}0.67 \\
0.25 \\
0.56\end{array}$ & $\begin{array}{l}1.04 \\
2.09 \\
2.46\end{array}$ & D & $\begin{array}{l}\mathrm{E} \\
\mathrm{Sa} \\
\mathrm{Sm}\end{array}$ & $\begin{array}{l}-2-7-73 \\
-2-7-74 \\
-2-7-75\end{array}$ & $\begin{array}{l}14.21 \\
15.68 \\
15.39\end{array}$ & $\begin{array}{l}4285 \pm 34 \\
4246 \pm 39 \\
4160 \pm 41\end{array}$ \\
\hline $\begin{array}{l}20 \mathrm{~A} \\
20 \mathrm{~B} \\
20 \mathrm{C}\end{array}$ & $\begin{array}{l}\vec{t} \\
+\end{array}$ & $\begin{array}{llllll}03 & 28 & 29 & -48 & 01 & 54 \\
03 & 29 & 15 & -48 & 08 & 07 \\
03 & 29 & 18 & -48 & 08 & 54\end{array}$ & $\begin{array}{l}0.63 \\
0.77 \\
0.95\end{array}$ & $\begin{array}{l}0.44 \\
0.66 \\
0.66\end{array}$ & $\begin{array}{c}9.88 \\
0.94 \\
10.79\end{array}$ & $\mathrm{D}$ & $\begin{array}{l}\text { So-a } \\
\text { Sbc } \\
\text { Sbc }\end{array}$ & $\begin{array}{l}200-29 \\
200-32 \\
200-33\end{array}$ & $\begin{array}{l}14.16 \\
15.54 \\
13.70\end{array}$ & $\begin{array}{r}6635 \pm 62 \\
13800 \pm 190 \\
6964 \pm 213\end{array}$ \\
\hline $\begin{array}{l}21 \mathrm{~A} \\
21 \mathrm{~B} \\
21 \mathrm{C}\end{array}$ & $\begin{array}{l}+ \\
+\end{array}$ & $\begin{array}{llllll}03 & 34 & 09 & -25 & 46 & 12 \\
03 & 34 & 17 & -25 & 49 & 36 \\
03 & 34 & 19 & -25 & 49 & 30\end{array}$ & $\begin{array}{l}1.36 \\
0.63 \\
0.90\end{array}$ & $\begin{array}{l}0.35 \\
0.17 \\
0.35\end{array}$ & $\begin{array}{l}3.84 \\
0.46 \\
4.00\end{array}$ & $D$ & $\begin{array}{l}\text { Sbc } \\
\text { So } \\
\text { Sb }\end{array}$ & $482-11$ & 15.21 & \\
\hline $\begin{array}{l}22 \mathrm{~A} \\
22 \mathrm{~B} \\
22 \mathrm{C}\end{array}$ & $\begin{array}{l}+ \\
+ \\
+\end{array}$ & $\begin{array}{llllll}03 & 52 & 17 & -20 & 34 & 24 \\
03 & 52 & 27 & -20 & 38 & 54 \\
03 & 52 & 52 & -20 & 31 & 42\end{array}$ & $\begin{array}{l}0.84 \\
1.57 \\
1.12\end{array}$ & $\begin{array}{l}0.45 \\
1.01 \\
1.01\end{array}$ & $\begin{array}{l}5.06 \\
9.27 \\
8.62\end{array}$ & $\mathrm{~T}$ & $\begin{array}{l}\mathrm{E} \\
\mathrm{Sa} \\
\mathrm{Sc}\end{array}$ & $\begin{array}{l}-3-10-53 \\
-3-10-54 \\
-3-10-55\end{array}$ & $\begin{array}{l}14.52 \\
13.14 \\
15.51\end{array}$ & $1859 \pm 8$ \\
\hline $\begin{array}{l}23 \mathrm{~A} \\
23 \mathrm{~B} \\
23 \mathrm{C}\end{array}$ & $\begin{array}{l}+ \\
+ \\
+\end{array}$ & $\begin{array}{llllll}03 & 59 & 23 & -67 & 46 & 30 \\
04 & 00 & 08 & -67 & 56 & 48 \\
04 & 00 & 42 & -67 & 45 & 00\end{array}$ & $\begin{array}{l}4.34 \\
1.99 \\
2.17\end{array}$ & $\begin{array}{l}1.58 \\
0.35 \\
0.27\end{array}$ & $\begin{array}{c}11.14 \\
12.22 \\
7.61\end{array}$ & $\mathrm{~T}$ & $\begin{array}{l}\text { Sa } \\
\text { SBa } \\
\text { SBcd }\end{array}$ & $\begin{array}{l}55-4 \\
55-5 \\
55-6\end{array}$ & $\begin{array}{l}11.85 \\
14.16 \\
15.06\end{array}$ & $\begin{array}{r}1341 \pm 14 \\
1323 \pm 8\end{array}$ \\
\hline $\begin{array}{l}24 \mathrm{~A} \\
24 \mathrm{~B} \\
24 \mathrm{C}\end{array}$ & $\begin{array}{l}+ \\
+ \\
+\end{array}$ & $\begin{array}{lllllll}04 & 13 & 47 & -40 & 48 & 19 \\
04 & 13 & 52 & -40 & 47 & 08 \\
04 & 13 & 57 & -40 & 46 & 15\end{array}$ & $\begin{array}{l}0.54 \\
0.45 \\
1.18\end{array}$ & $\begin{array}{l}0.44 \\
0.27 \\
0.53\end{array}$ & $\begin{array}{l}1.48 \\
1.28 \\
2.78\end{array}$ & L & $\begin{array}{l}\mathrm{E} \\
\mathrm{S}(\mathrm{r}) \\
\mathrm{Sc}\end{array}$ & $303-6$ & 15.86 & \\
\hline
\end{tabular}




\begin{tabular}{|c|c|c|c|c|c|c|c|c|c|c|}
\hline$\frac{\mathrm{TS}}{1}$ & $\frac{\text { Is. }}{2}$ & $\frac{\text { R.A.(1950) Dec. }}{3}$ & $\frac{a}{4}$ & $\frac{b}{5}$ & $\frac{X_{i j}}{6}$ & $\frac{C .}{7}$ & $\frac{\text { Type }}{8}$ & $\frac{\text { Alias. }}{9}$ & $\begin{array}{l}B_{t} \\
10\end{array}$ & $\frac{v \pm \sigma}{11}$ \\
\hline $\begin{array}{l}25 \mathrm{~A} \\
25 \mathrm{~B} \\
25 \mathrm{C}\end{array}$ & $\begin{array}{l}+ \\
+ \\
+\end{array}$ & $\begin{array}{llllll}04 & 26 & 34 & -48 & 01 & 12 \\
04 & 26 & 56 & -47 & 55 & 30 \\
04 & 27 & 08 & -47 & 53 & 24\end{array}$ & $\begin{array}{l}1.63 \\
1.45 \\
1.27\end{array}$ & $\begin{array}{l}1.40 \\
1.05 \\
0.74\end{array}$ & $\begin{array}{l}6.78 \\
2.91 \\
9.66\end{array}$ & D & $\begin{array}{l}\text { Spec } \\
\text { E } \\
\text { SBc }\end{array}$ & $\begin{array}{l}202-23 \\
202-25 \\
202-26\end{array}$ & $\begin{array}{l}13.47 \\
13.68 \\
13.81\end{array}$ & $\begin{array}{l}4913 \pm 61 \\
4696 \pm 53 \\
5105 \pm 29\end{array}$ \\
\hline $\begin{array}{l}26 \mathrm{~A} \\
26 \mathrm{~B} \\
26 \mathrm{C}\end{array}$ & $\begin{array}{l}+ \\
+ \\
+\end{array}$ & $\begin{array}{llllll}04 & 37 & 01 & -24 & 16 & 54 \\
04 & 37 & 06 & -24 & 16 & 42 \\
04 & 38 & 09 & -24 & 24 & 54\end{array}$ & $\begin{array}{l}1.08 \\
1.54 \\
1.08\end{array}$ & $\begin{array}{l}0.44 \\
0.13 \\
0.96\end{array}$ & $\begin{array}{r}1.13 \\
16.53 \\
17.44\end{array}$ & D & $\begin{array}{l}\mathrm{Sc} \\
\mathrm{Sc} \\
\mathrm{SBc}\end{array}$ & $\begin{array}{l}485-3 \\
485-4 \\
485-6\end{array}$ & $\begin{array}{l}14.53 \\
15.99 \\
14.58\end{array}$ & $\begin{array}{l}4486 \pm 77 \\
4409 \pm 47 \\
4422 \pm 42\end{array}$ \\
\hline $\begin{array}{l}27 \mathrm{~A} \\
27 \mathrm{~B} \\
27 \mathrm{C}\end{array}$ & $\begin{array}{l}\overrightarrow{+} \\
+\end{array}$ & $\begin{array}{llllll}04 & 49 & 41 & -61 & 19 & 54 \\
04 & 49 & 50 & -61 & 25 & 42 \\
04 & 50 & 03 & -61 & 26 & 18\end{array}$ & $\begin{array}{l}0.98 \\
1.27 \\
1.18\end{array}$ & $\begin{array}{l}0.74 \\
1.09 \\
0.61\end{array}$ & $\begin{array}{l}5.90 \\
1.66 \\
6.92\end{array}$ & D & $\begin{array}{l}\text { Sc } \\
\text { SBc } \\
\text { Scd }\end{array}$ & $\begin{array}{l}119-12 \\
119-13 \\
119-14\end{array}$ & $\begin{array}{l}14.56 \\
13.67 \\
15.06\end{array}$ & $\begin{array}{l}5941 \pm 60 \\
5903 \pm 40\end{array}$ \\
\hline $\begin{array}{l}28 \mathrm{~A} \\
28 \mathrm{~B} \\
28 \mathrm{C}\end{array}$ & $\begin{array}{l}+ \\
+ \\
+\end{array}$ & $\begin{array}{llllll}04 & 56 & 56 & -11 & 11 & 35 \\
04 & 57 & 02 & -11 & 12 & 24 \\
04 & 57 & 07 & -11 & 11 & 49\end{array}$ & $\begin{array}{l}3.25 \\
1.23 \\
1.79\end{array}$ & $\begin{array}{l}1.68 \\
1.01 \\
0.67\end{array}$ & $\begin{array}{l}1.67 \\
1.34 \\
2.69\end{array}$ & $\mathrm{~L}$ & $\begin{array}{l}\text { SBb } \\
\text { S0 } \\
\text { Sa }\end{array}$ & $\begin{array}{l}-2-13-27 \\
-2-13-28 \\
-2-13-30\end{array}$ & $\begin{array}{l}13.15 \\
13.70 \\
14.76\end{array}$ & $\begin{array}{l}4607 \pm 134 \\
3998 \pm 122 \\
4301 \pm 291\end{array}$ \\
\hline $\begin{array}{l}29 \mathrm{~A} \\
29 \mathrm{~B} \\
29 \mathrm{C}\end{array}$ & $\begin{array}{l}+ \\
+ \\
-\end{array}$ & $\begin{array}{llllll}04 & 58 & 52 & -75 & 29 & 48 \\
04 & 58 & 58 & -75 & 31 & 12 \\
04 & 59 & 16 & -75 & 31 & 58\end{array}$ & $\begin{array}{l}1.99 \\
0.95 \\
0.45\end{array}$ & $\begin{array}{l}0.96 \\
0.52 \\
0.27\end{array}$ & $\begin{array}{l}1.45 \\
1.35 \\
2.62\end{array}$ & L & $\begin{array}{l}\text { SBbc } \\
\text { Sab } \\
\text { Pec }\end{array}$ & $\begin{array}{l}33-4 \\
33-5\end{array}$ & $\begin{array}{l}14.04 \\
14.21\end{array}$ & $5192 \pm 60$ \\
\hline $\begin{array}{l}30 \mathrm{~A} \\
30 \mathrm{~B} \\
30 \mathrm{C}\end{array}$ & $\begin{array}{l}+ \\
+ \\
-\end{array}$ & $\begin{array}{llllll}05 & 14 & 00 & -62 & 13 & 34 \\
05 & 14 & 02 & -62 & 17 & 00 \\
05 & 14 & 22 & -62 & 14 & 03\end{array}$ & $\begin{array}{l}1.04 \\
1.08 \\
0.82\end{array}$ & $\begin{array}{l}0.27 \\
0.66 \\
0.35\end{array}$ & $\begin{array}{l}3.44 \\
3.76 \\
2.60\end{array}$ & $\mathbf{T}$ & $\begin{array}{l}\mathrm{Sb} \\
\mathrm{SBb} \\
\mathrm{SO}-\mathrm{a}\end{array}$ & $\begin{array}{l}119-46 \\
119-47\end{array}$ & $\begin{array}{l}14.95 \\
13.98\end{array}$ & $\begin{array}{l}4966 \pm 60 \\
5100 \pm 63\end{array}$ \\
\hline $\begin{array}{l}31 \mathrm{~A} \\
31 \mathrm{~B} \\
31 \mathrm{C}\end{array}$ & $\begin{array}{l}+ \\
+ \\
-\end{array}$ & $\begin{array}{llllll}05 & 14 & 03 & -53 & 47 & 36 \\
05 & 15 & 06 & -54 & 09 & 30 \\
05 & 16 & 05 & -54 & 07 & 12\end{array}$ & $\begin{array}{l}2.54 \\
2.17 \\
1.27\end{array}$ & $\begin{array}{l}2.02 \\
1.31 \\
0.61\end{array}$ & $\begin{array}{c}23.79 \\
8.94 \\
26.59\end{array}$ & $\mathrm{D}$ & $\begin{array}{l}\text { SBbc } \\
\text { SO } \\
\text { SO-a }\end{array}$ & $\begin{array}{l}159-2 \\
159-3 \\
159-4\end{array}$ & $\begin{array}{l}13.71 \\
13.97 \\
14.45\end{array}$ & $\begin{array}{r}4290 \pm 8 \\
3902 \pm 60 \\
10300 \pm 190\end{array}$ \\
\hline $\begin{array}{l}32 \mathrm{~A} \\
32 \mathrm{~B} \\
32 \mathrm{C}\end{array}$ & $\begin{array}{l}+ \\
+ \\
+\end{array}$ & $\begin{array}{llllll}06 & 18 & 56 & -20 & 01 & 24 \\
06 & 19 & 36 & -20 & 06 & 06 \\
06 & 19 & 41 & -20 & 12 & 00\end{array}$ & $\begin{array}{l}3.71 \\
1.18 \\
1.53\end{array}$ & $\begin{array}{l}1.31 \\
0.52 \\
1.49\end{array}$ & $\begin{array}{c}10.50 \\
6.01 \\
14.96\end{array}$ & $\mathbf{T}$ & $\begin{array}{l}\text { SBa } \\
\text { Sbc } \\
\text { SBm }\end{array}$ & $\begin{array}{l}556-15 \\
556-18 \\
556-19\end{array}$ & $\begin{array}{l}12.71 \\
15.80 \\
14.96\end{array}$ & $\begin{array}{r}1981 \pm 11 \\
1868 \pm 8\end{array}$ \\
\hline $\begin{array}{l}33 \mathrm{~A} \\
33 \mathrm{~B} \\
33 \mathrm{C}\end{array}$ & $\begin{array}{l}+ \\
+ \\
+\end{array}$ & $\begin{array}{llllll}06 & 19 & 26 & -57 & 33 & 12 \\
06 & 19 & 27 & -57 & 30 & 24 \\
06 & 19 & 40 & -57 & 28 & 18\end{array}$ & $\begin{array}{l}1.90 \\
1.08 \\
1.27\end{array}$ & $\begin{array}{l}0.52 \\
0.35 \\
0.52\end{array}$ & $\begin{array}{l}2.80 \\
2.93 \\
5.25\end{array}$ & $\mathrm{~L}$ & $\begin{array}{l}\mathrm{Sa} \\
\text { SBa } \\
\text { SBd }\end{array}$ & $\begin{array}{l}121-24 \\
121-25 \\
161-1\end{array}$ & $\begin{array}{l}13.79 \\
14.14 \\
14.74\end{array}$ & $\begin{array}{l}2442 \pm 96 \\
2601 \pm 47\end{array}$ \\
\hline $\begin{array}{l}34 \mathrm{~A} \\
34 \mathrm{~B} \\
34 \mathrm{C}\end{array}$ & $\begin{array}{l}+ \\
+ \\
+\end{array}$ & $\begin{array}{llllll}08 & 17 & 48 & -67 & 25 & 12 \\
08 & 18 & 03 & -67 & 27 & 12 \\
08 & 18 & 37 & -67 & 20 & 36\end{array}$ & $\begin{array}{l}1.27 \\
1.08 \\
1.36\end{array}$ & $\begin{array}{l}0.74 \\
0.61 \\
0.70\end{array}$ & $\begin{array}{l}2.46 \\
7.35 \\
6.58\end{array}$ & $\mathrm{~T}$ & $\begin{array}{l}\mathrm{SBc} \\
\mathrm{E} \\
\mathrm{E}\end{array}$ & $\begin{array}{l}89-15 \\
89-16 \\
89-17\end{array}$ & $\begin{array}{l}14.31 \\
14.39 \\
13.67\end{array}$ & \\
\hline $\begin{array}{l}35 \mathrm{~A} \\
35 \mathrm{~B} \\
35 \mathrm{C}\end{array}$ & $\begin{array}{l}+ \\
+ \\
+\end{array}$ & $\begin{array}{llllll}08 & 24 & 44 & -77 & 41 & 18 \\
08 & 24 & 52 & -77 & 39 & 18 \\
08 & 25 & 41 & -77 & 37 & 00\end{array}$ & $\begin{array}{l}1.36 \\
0.99 \\
0.82\end{array}$ & $\begin{array}{l}0.61 \\
0.44 \\
0.79\end{array}$ & $\begin{array}{l}2.04 \\
3.48 \\
5.26\end{array}$ & $\mathrm{~L}$ & $\begin{array}{l}\text { SBbc } \\
\text { Sc } \\
\mathrm{Sb}\end{array}$ & $\begin{array}{l}18-7 \\
18-8 \\
18-9\end{array}$ & $\begin{array}{l}13.52 \\
14.40 \\
13.80\end{array}$ & $\begin{array}{l}5291 \pm 87 \\
5432 \pm 87 \\
5313 \pm 27\end{array}$ \\
\hline $\begin{array}{l}36 \mathrm{~A} \\
36 \mathrm{~B} \\
36 \mathrm{C}\end{array}$ & $\begin{array}{l}+ \\
+ \\
+\end{array}$ & $\begin{array}{llllll}08 & 54 & 27 & -20 & 21 & 54 \\
08 & 54 & 40 & -20 & 20 & 06 \\
08 & 54 & 55 & -20 & 23 & 00\end{array}$ & $\begin{array}{l}1.36 \\
1.18 \\
1.36\end{array}$ & $\begin{array}{l}0.52 \\
0.79 \\
0.79\end{array}$ & $\begin{array}{l}3.53 \\
4.55 \\
6.64\end{array}$ & $\mathrm{~T}$ & $\begin{array}{l}\mathrm{Sm} \\
\mathrm{Sab} \\
\mathrm{Sa}\end{array}$ & $\begin{array}{l}563-33 \\
563-34 \\
563-36\end{array}$ & $\begin{array}{l}14.40 \\
13.86 \\
13.61\end{array}$ & 2630 上 60 \\
\hline
\end{tabular}




\begin{tabular}{|c|c|c|c|c|c|c|c|c|c|c|}
\hline TS & $\frac{\text { Is. }}{2}$ & R.A. $(1950)$ Dec. & $a$ & $\frac{b}{5}$ & $X_{i j}$ & C. & Type & Alias. & $\overline{B_{t}}$ & $v \pm \sigma$ \\
\hline 1 & 2 & 3 & 4 & 5 & 6 & 7 & 8 & $\overline{9}$ & 10 & 11 \\
\hline $37 \mathrm{~A}$ & - & $\begin{array}{llllll}09 & 52 & 12 & -32 & 52 & 48\end{array}$ & 1.45 & 0.27 & 9.74 & \multirow[t]{3}{*}{ D } & $\mathrm{Sb}$ & $374-8$ & 15.62 & $1510 \pm 60$ \\
\hline $37 \mathrm{~B}$ & + & $095258-325400$ & 3.34 & 1.84 & 4.40 & & $\mathrm{Sb}$ & $374-10$ & 12.6 & 3012 \\
\hline $37 \mathrm{C}$ & + & 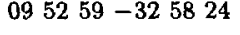 & 1.53 & 0.78 & 11.34 & & SBbc & $374-11$ & 13.65 & $2601 \pm 44$ \\
\hline $38 \mathrm{~A}$ & + & $100336-155246$ & 2.35 & 1.01 & 6.75 & \multirow[t]{3}{*}{$\mathrm{T}$} & $\mathrm{Sb}$ & $-3-26-20$ & 14.31 & $4635 \pm 10$ \\
\hline $38 B$ & + & $100350-154654$ & 2.35 & 0.34 & 6.49 & & Sc & $-3-26-21$ & 14.89 & $5009 \pm 7$ \\
\hline $38 \mathrm{C}$ & + & $100401-155255$ & 1.57 & 0.31 & 5.77 & & $\mathrm{Sb}$ & $-3-26-22$ & 14.62 & $4536 \pm 43$ \\
\hline $39 \mathrm{~A}$ & + & $103037-070555$ & 0.95 & 0.50 & 0.58 & \multirow[t]{3}{*}{$\mathrm{D}$} & Sc & $-1-27-16$ & 15.11 & \\
\hline $39 \mathrm{~B}$ & + & $\begin{array}{llllll}10 & 30 & 39 & -07 & 05 & 37\end{array}$ & 0.90 & 0.67 & 6.87 & & $\mathrm{Sa}$ & $-1-27-17$ & 15.57 & \\
\hline $39 \mathrm{C}$ & + & $103043-071225$ & 1.01 & 1.01 & 6.67 & & So & $-1-27-18$ & 14.02 & $4942 \pm 44$ \\
\hline $40 \mathrm{~A}$ & - & $\begin{array}{llllll}10 & 36 & 55 & -30 & 02 & 18\end{array}$ & 3.34 & 1.05 & 11.24 & \multirow[t]{3}{*}{$\mathbf{T}$} & SBbc & $437-30$ & 13.73 & $3773 \pm 15$ \\
\hline $40 \mathrm{~B}$ & + & 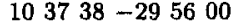 & 1.63 & 1.05 & 4.59 & & SBa & $437-33$ & 13.72 & $3338 \pm 148$ \\
\hline $40 \mathrm{C}$ & + & $103744-300024$ & 1.81 & 0.79 & 10.78 & & $\mathrm{SBbc}$ & $437-35$ & 14.26 & $3445 \pm 65$ \\
\hline $41 \mathrm{~A}$ & - & 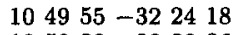 & 2.35 & 2.20 & 17.18 & \multirow[t]{3}{*}{$\mathbf{L}$} & SBab & $437-67$ & 13.60 & $3162 \pm 25$ \\
\hline $41 B$ & + & $\begin{array}{llllll}10 & 50 & 32 & -32 & 39 & 36\end{array}$ & 3.53 & 1.05 & 19.42 & & SBab & $376-25$ & 12.83 & $3290 \pm 15$ \\
\hline $41 \mathrm{C}$ & + & $\begin{array}{lllll}10 & 51 & 46 & -3251 & 11\end{array}$ & 1.81 & 1.05 & 35.67 & & $\mathrm{SO}-\mathrm{a}$ & $376-26$ & 13.52 & $3478 \pm 149$ \\
\hline $42 \mathrm{~A}$ & + & $111425-255136$ & 1.27 & 0.17 & 12.97 & \multirow[t]{3}{*}{$\mathbf{T}$} & Sbc & $503-8$ & 15.81 & \\
\hline $42 B$ & + & 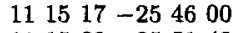 & 1.72 & 0.96 & 5.86 & & $\mathrm{SBC}$ & $503-11$ & 15.55 & $2047 \pm 9$ \\
\hline $42 \mathrm{C}$ & + & $\begin{array}{llllll}11 & 15 & 23 & -25 & 51 & 42\end{array}$ & 1.63 & 1.14 & 13.05 & & $\mathrm{E}$ & $503-12$ & 13.60 & $2205 \pm 42$ \\
\hline $43 \mathrm{~A}$ & + & 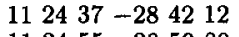 & 2.08 & 1.75 & 17.25 & \multirow[t]{3}{*}{ D } & S0-a & $439-8$ & 13.21 & $1636 \pm 30$ \\
\hline $43 \mathrm{~B}$ & + & $\begin{array}{llllll}1124 & 55 & -28 & 59 & 00\end{array}$ & 1.53 & 0.44 & 5.28 & & Sa & 439- 9 & 14.79 & $7137 \pm 102$ \\
\hline $43 \mathrm{C}$ & + & $112504-285406$ & 1.72 & 0.66 & 13.28 & & $\mathrm{Sb}$ & $439-10$ & 15.77 & $7164 \pm 34$ \\
\hline $44 \mathrm{~A}$ & + & $121208-351354$ & 2.99 & 1.40 & 5.77 & \multirow[t]{3}{*}{$\mathrm{T}$} & $\mathrm{SBb}$ & $380-1$ & 12.90 & $2689 \pm 6$ \\
\hline $44 B$ & + & $\begin{array}{llllll}12 & 12 & 18 & -35 & 19 & 18\end{array}$ & 1.18 & 0.44 & 8.14 & & Sbc & $380-2$ & 15.30 & $2626 \pm 44$ \\
\hline $44 \mathrm{C}$ & + & $121257-352106$ & 3.53 & 1.40 & 12.31 & & $\mathrm{Sb}$ & $380-6$ & 12.63 & $2943 \pm 8$ \\
\hline $45 \mathrm{~A}$ & + & $123759-362755$ & 1.36 & 0.13 & 2.65 & \multirow[t]{3}{*}{$\mathrm{T}$} & & $381-6$ & 15.93 & $3180 \pm 60$ \\
\hline $45 \mathrm{~B}$ & + & $123811-362900$ & 1.08 & 0.96 & 1.79 & & SBbc & 381- 8 & 13.19 & $3396 \pm 160$ \\
\hline $45 \mathrm{C}$ & + & $123816-362730$ & 1.45 & 1.14 & 3.44 & & $\mathrm{SBbc}$ & $381-9$ & 13.93 & $3302 \pm 71$ \\
\hline $46 \mathrm{~A}$ & + & $124757-091046$ & 1.01 & 0.56 & 4.16 & \multirow[t]{3}{*}{$\mathrm{D}$} & So & $-1-33-21$ & 13.74 & $4645 \pm 109$ \\
\hline $46 \mathrm{~B}$ & + & $124758-091453$ & 1.40 & 0.67 & 3.43 & & Scd & $-1-33-22$ & 14.62 & \\
\hline $46 \mathrm{C}$ & + & $124758-091128$ & 1.57 & 0.34 & 0.76 & & $\mathrm{SBa}$ & $-1-33-23$ & 15 & $4515 \pm 96$ \\
\hline $47 \mathrm{~A}$ & + & $133700-504700$ & 0.99 & 0.83 & 6.45 & \multirow[t]{3}{*}{$\mathrm{T}$} & Sbc & $220-27$ & 14.02 & $4045 \pm 49$ \\
\hline $47 \mathrm{~B}$ & + & $133705-505324$ & 1.90 & 0.24 & 5.15 & & $\mathrm{Sbc}$ & $220-28$ & 14.73 & $3639 \pm 10$ \\
\hline $47 \mathrm{C}$ & + & 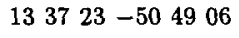 & 0.82 & 0.70 & 4.19 & & $\mathrm{SO}$ & $220-29$ & 13.37 & $4031 \pm 106$ \\
\hline $48 \mathrm{~A}$ & + & $134622-065654$ & 2.58 & 0.45 & 3.43 & \multirow[t]{3}{*}{$\mathrm{D}$} & $\mathrm{Sb}$ & $-1-35-13$ & 15.07 & $7644 \pm 41$ \\
\hline $48 \mathrm{~B}$ & + & $134634-065835$ & 1.34 & 0.78 & 0.93 & & SO & $-1-35-14$ & 13.10 & $7246 \pm 36$ \\
\hline $48 \mathrm{C}$ & - & $\begin{array}{llllll}13 & 46 & 35 & -06 & 57 & 41\end{array}$ & 078 & 0.34 & 3.31 & & $\mathrm{Sb}$ & $-1-35-15$ & 15.66 & $7368 \pm 104$ \\
\hline
\end{tabular}




\begin{tabular}{|c|c|c|c|c|c|c|c|c|c|c|}
\hline TS & Is. & R.A.(1950) Dec. & $a$ & $b$ & $X_{i j}$ & C. & Type & Alias. & $\overline{B_{t}}$ & $v \pm \sigma$ \\
\hline 1 & 2 & 3 & 4 & 5 & 6 & 7 & 8 & 9 & 10 & 11 \\
\hline $49 \mathrm{~A}$ & + & $\begin{array}{llllll}13 & 47 & 35 & -37 & 02 & 30\end{array}$ & 2.44 & 0.27 & 22.71 & \multirow[t]{3}{*}{$\mathbf{T}$} & SBcd & 383-91 & 14.35 & $1079 \pm 8$ \\
\hline $49 B$ & - & 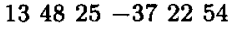 & 1.72 & 0.27 & 14.87 & & $\mathrm{Sb}$ & $384-3$ & 14.37 & $3849 \pm 129$ \\
\hline $49 \mathrm{C}$ & + & $\begin{array}{llllll}13 & 49 & 19 & -37 & 12 & 36\end{array}$ & 2.26 & 0.22 & 23.04 & & SBd & $384-5$ & 15.41 & $1640 \pm 8$ \\
\hline $50 \mathrm{~A}$ & + & $142100-28 \quad 2742$ & 1.36 & 0.70 & 7.32 & \multirow[t]{3}{*}{$\mathrm{T}$} & SBbc & $446-58$ & 13.50 & \multirow[t]{3}{*}{$4347 \pm 10$} \\
\hline $50 \mathrm{~B}$ & + & 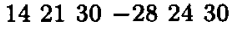 & 1.27 & 0.30 & 5.61 & & Sbc & $446-59$ & 15.28 & \\
\hline $50 \mathrm{C}$ & + & $\begin{array}{llllll}14 & 21 & 37 & -28 & 29 & 54\end{array}$ & 1.04 & 0.16 & 8.42 & & Sbc & $446-60$ & 16.21 & \\
\hline $51 \mathrm{~A}$ & + & $144433-22 \quad 1154$ & 1.08 & 0.70 & 14.98 & \multirow[t]{3}{*}{$\mathrm{L}$} & Scd & $580-25$ & 14.34 & \\
\hline $51 \mathrm{~B}$ & + & $144436-215656$ & 1.36 & 0.79 & 7.26 & & So & $580-26$ & 14.92 & $3194 \pm 133$ \\
\hline $51 \mathrm{C}$ & + & $144437-22 \quad 0412$ & 1.90 & 1.49 & 7.55 & & $\mathrm{SBa}$ & $580-27$ & 13.66 & $3286 \pm 9$ \\
\hline $52 \mathrm{~A}$ & + & $144648-095758$ & 2.80 & 1.12 & 0.80 & \multirow[t]{3}{*}{$\mathrm{D}$} & Sed & $-2-38-16$ & 13.38 & \multirow{3}{*}{$\begin{array}{l}1859 \pm 20 \\
1856 \pm 10\end{array}$} \\
\hline $52 \mathrm{~B}$ & + & $144650-095719$ & 1.90 & 0.56 & 5.45 & & Sdm & $-2-38-17$ & 14.28 & \\
\hline $53 \mathrm{C}$ & + & $144653-095155$ & 0.90 & 0.34 & 6.15 & & Irr & & & \\
\hline $53 \mathrm{~A}$ & + & $\begin{array}{llllll}14 & 58 & 20 & -37 & 47 & 48\end{array}$ & 2.26 & 0.44 & 3.87 & \multirow[t]{3}{*}{$\mathrm{T}$} & $\mathrm{Sa}$ & $328-5$ & 15.0 & \multirow{3}{*}{$4434 \pm 60$} \\
\hline $53 \mathrm{~B}$ & + & $1458 \quad 33-37 \quad 4454$ & 0.90 & 0.79 & 3.87 & & $\mathrm{SBc}$ & $328-6$ & 14.58 & \\
\hline $53 \mathrm{C}$ & + & $\begin{array}{llllll}14 & 58 & 51 & -37 & 46 & 24\end{array}$ & 1.36 & 0.22 & 6.28 & & Sbc & 328- 7 & 14.76 & \\
\hline $54 \mathrm{~A}$ & + & $\begin{array}{llllll}17 & 06 & 19 & -77 & 28 & 30\end{array}$ & 3.71 & 2.20 & 6.69 & \multirow[t]{3}{*}{$\mathbf{L}$} & Sc & 44- 3 & 12.40 & \multirow{3}{*}{$\begin{array}{l}2941 \pm 7 \\
2948 \pm 8\end{array}$} \\
\hline 54B & + & $\begin{array}{llllll}17 & 08 & 12 & -7 & 25 & 54\end{array}$ & 3.26 & 0.61 & 17.15 & & $\mathrm{SBb}$ & 44- 5 & 13.94 & \\
\hline $54 \mathrm{C}$ & + & 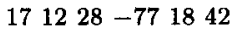 & 1.72 & 0.30 & 22.52 & & SBcd & $44-10$ & 14.61 & \\
\hline $55 \mathrm{~A}$ & + & 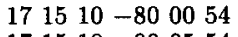 & 1.18 & 1.05 & 5.01 & \multirow[t]{3}{*}{$\mathrm{T}$} & SBcd & 24- 1 & \multirow{3}{*}{13.80} & $4845 \pm 60$ \\
\hline $55 B$ & + & $\begin{array}{llllll}17 & 15 & 18 & -80 & 05 & 54\end{array}$ & 1.27 & 1.23 & 5.56 & & Sc & 24- 2 & & $5330 \pm 60$ \\
\hline $55 \mathrm{C}$ & + & 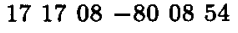 & 1.36 & 0.52 & 9.47 & & So & 24- 5 & & $4872 \pm 60$ \\
\hline $56 \mathrm{~A}$ & - & $\begin{array}{llllll}17 & 23 & 33 & -85 & 16 & 30\end{array}$ & 2.26 & 1.49 & 51.61 & \multirow[t]{3}{*}{$\mathrm{D}$} & Sbc & $9-10$ & & $2422 \pm 8$ \\
\hline $56 \mathrm{~B}$ & + & 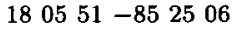 & 1.18 & 0.88 & 0.58 & & So & $10-1$ & 12.13 & $2437 \pm 21$ \\
\hline $56 \mathrm{C}$ & + & $\begin{array}{llllll}18 & 06 & 18 & -85 & 25 & 18\end{array}$ & 2.08 & 0.79 & 53.18 & & $\operatorname{Irr}$ & $10-2$ & 12.39 & $2515 \pm 9$ \\
\hline $57 \mathrm{~A}$ & + & $\begin{array}{llllll}18 & 09 & 19 & -57 & 44 & 48\end{array}$ & 0.32 & 0.30 & 0.42 & \multirow[t]{3}{*}{$\mathbf{L}$} & Pec & $140-9$ & 14.77 & \multirow{6}{*}{$\begin{array}{r}4960 \pm 62 \\
5189 \pm 35 \\
5140 \pm 156\end{array}$} \\
\hline $57 \mathrm{~B}$ & + & $180920-574424$ & 1.63 & 1.05 & 1.41 & & $\mathrm{Sb}$ & $140-10$ & 14.28 & \\
\hline $57 \mathrm{C}$ & + & $\begin{array}{llllll}18 & 09 & 21 & -57 & 45 & 48\end{array}$ & 0.82 & 0.30 & 1.04 & & $\mathrm{Sa}$ & $140-11$ & 15.00 & \\
\hline $58 \mathrm{~A}$ & + & $\begin{array}{llllll}18 & 31 & 32 & -67 & 16 & 23\end{array}$ & 0.54 & 0.48 & 0.87 & \multirow[t]{3}{*}{$\mathbf{L}$} & $\mathrm{SBa}$ & & & \\
\hline $58 \mathrm{~B}$ & + & $\begin{array}{llllll}18 & 31 & 37 & -67 & 17 & 06\end{array}$ & 1.08 & 0.44 & 2.13 & & $\mathrm{Sb}$ & $103-29$ & 15.34 & \\
\hline $58 \mathrm{C}$ & + & $\begin{array}{llll}1831 & 47 & -67 & 1900\end{array}$ & 1.08 & 0.27 & 3.00 & & Sbc & $103-30$ & 15.65 & \\
\hline $59 \mathrm{~A}$ & + & $\begin{array}{llllll}19 & 13 & 57 & -60 & 35 & 30\end{array}$ & 1.90 & 1.14 & 1.85 & \multirow[t]{3}{*}{$\mathrm{T}$} & $\mathrm{SBb}$ & $141-48$ & 12.57 & \multirow{3}{*}{$\begin{array}{r}3810 \pm 119 \\
3834 \pm 96 \\
4221 \pm 76\end{array}$} \\
\hline $59 \mathrm{~B}$ & + & $\begin{array}{llllll}19 & 14 & 12 & -60 & 35 & 18\end{array}$ & 2.44 & 2.19 & 2.91 & & SBb & $141-49$ & 12.76 & \\
\hline $59 \mathrm{C}$ & - & $\begin{array}{llllll}19 & 14 & 14 & -60 & 38 & 12\end{array}$ & 2.35 & 0.44 & 3.41 & & So-a & $141-50$ & 13.57 & \\
\hline $60 \mathrm{~A}$ & + & $\begin{array}{llllll}19 & 26 & 46 & -39 & 30 & 49\end{array}$ & 0.73 & 0.39 & 0.68 & \multirow[t]{3}{*}{$\mathrm{T}$} & Sab\} & 338- 8$\}$ & 16.73 & \multirow[t]{3}{*}{\} $2835 \pm 67$} \\
\hline $60 \mathrm{~B}$ & + & $\begin{array}{llllll}19 & 26 & 49 & -39 & 31 & 09\end{array}$ & 0.45 & 0.24 & 0.24 & & $\mathrm{Pec}$ & & & \\
\hline $60 \mathrm{C}$ & + & $\begin{array}{llllll}19 & 26 & 50 & -39 & 31 & 01\end{array}$ & 0.73 & 0.27 & 0.80 & & Pec & & & \\
\hline
\end{tabular}




\begin{tabular}{|c|c|c|c|c|c|c|c|c|c|c|}
\hline TS & Is. & R.A.(1950) Dec. & $\bar{a}$ & $b$ & $X_{i j}$ & C. & Type & Alias. & $\overline{B_{t}}$ & $v \pm \sigma$ \\
\hline 1 & $\overline{2}$ & 3 & 4 & 5 & 6 & $\overline{7}$ & 8 & 9 & 10 & 11 \\
\hline $61 \mathrm{~A}$ & - & $195721-47 \quad 1322$ & 0.99 & 0.27 & 0.85 & $L$ & $\mathrm{Sa}$ & & & \\
\hline $61 B$ & + & $195721-47 \quad 1230$ & 1.90 & 0.61 & 1.50 & & $\mathrm{Sb}\}$ & $284-8\}$ & 13.83 & $6348 \pm 75$ \\
\hline $61 \mathrm{C}$ & + & $195729-471151$ & 1.08 & 0.57 & 2.02 & & Sbc & & & \\
\hline $62 \mathrm{~A}$ & + & $\begin{array}{llllll}20 & 27 & 52 & -25 & 31 & 54\end{array}$ & 0.77 & 0.13 & 0.49 & $\mathrm{D}$ & $\mathbf{S b}$ & $528-13$ & 15.57 & \\
\hline $62 \mathrm{~B}$ & + & $202754-253206$ & 0.82 & 0.27 & 3.76 & & $\mathrm{Sa}$ & $528-14$ & 15.24 & \\
\hline $62 \mathrm{C}$ & + & $202758-252827$ & 0.68 & 0.10 & 3.71 & & $\mathrm{Sb}$ & & & \\
\hline $63 \mathrm{~A}$ & + & $\begin{array}{llllll}20 & 45 & 19 & -20 & 02 & 27\end{array}$ & 0.63 & 0.44 & 1.45 & $\mathrm{~T}$ & E-SO & & & \\
\hline $63 \mathrm{~B}$ & + & $\begin{array}{llllll}20 & 45 & 19 & -20 & 01 & 00\end{array}$ & 0.86 & 0.27 & 1.37 & & $\mathrm{Sa}$ & & & \\
\hline $63 \mathrm{C}$ & + & $204523-200200$ & 1.36 & 0.24 & 1.04 & & Sb & $597-36$ & 15.22 & $8694 \pm 41$ \\
\hline $64 \mathrm{~A}$ & + & $205537-20 \quad 1030$ & 1.27 & 0.44 & 9.87 & $\mathrm{~L}$ & $\mathrm{Sb}$ & $598-9$ & 14.38 & \\
\hline $64 \mathrm{~B}$ & + & $\begin{array}{llllll}20 & 56 & 15 & -20 & 14 & 12\end{array}$ & 1.36 & 0.96 & 15.07 & & $\mathrm{Sc}$ & 598-11 & 15.74 & \\
\hline $64 \mathrm{C}$ & - & $205707 \quad-201922$ & 0.90 & 0.52 & 22.89 & & So & & & \\
\hline $65 \mathrm{~A}$ & + & $\begin{array}{llllll}21 & 41 & 35 & -49 & 14 & 10\end{array}$ & 0.82 & 0.48 & 0.95 & $\mathrm{D}$ & $\mathrm{Sm}$ & & & \\
\hline $65 B$ & + & $\begin{array}{llllll}21 & 41 & 39 & -49 & 14 & 51\end{array}$ & 0.63 & 0.35 & 3.15 & & E-SO & & & \\
\hline $65 \mathrm{C}$ & + & $214158-491418$ & 0.82 & 0.35 & 3.76 & & $\operatorname{Irr}$ & 236-39 & 15.08 & \\
\hline $66 \mathrm{~A}$ & + & $\begin{array}{llllll}21 & 59 & 09 & -32 & 12 & 54\end{array}$ & 1.18 & 0.79 & 1.27 & $\mathrm{~T}$ & $\mathrm{E}$ & $466-39$ & 12.86 & $2548 \pm 75$ \\
\hline $66 \mathrm{~B}$ & + & $\begin{array}{lllllllllll}21 & 59 & 12 & -32 & 14 & 00\end{array}$ & 1.36 & 0.61 & 0.43 & & Sab & $466-40$ & 13.85 & $2754 \pm 88$ \\
\hline $66 \mathrm{C}$ & + & $\begin{array}{llllll}21 & 59 & 14 & -32 & 13 & 54\end{array}$ & 1.08 & 0.70 & 1.44 & & $\mathrm{E}$ & $466-41$ & 12.39 & $2528 \pm 28$ \\
\hline $67 \mathrm{~A}$ & + & $221233-460600$ & 2.54 & 0.79 & 1.91 & $\mathrm{~T}$ & $\mathrm{SO}-\mathrm{a}$ & $289-7$ & 12.87 & $2152 \pm 8$ \\
\hline $67 \mathrm{~B}$ & + & $\begin{array}{llllll}22 & 12 & 44 & -46 & 05 & 48\end{array}$ & 1.53 & 1.05 & 4.26 & & $\mathrm{SBa}$ & 289- 8 & 13.10 & $1915 \pm 8$ \\
\hline $67 \mathrm{C}$ & + & $\begin{array}{llllll}22 & 12 & 48 & -46 & 01 & 48\end{array}$ & 1.72 & 1.40 & 4.94 & & $\mathrm{SBm}$ & 289- 9 & 13.87 & $2042 \pm 42$ \\
\hline $68 \mathrm{~A}$ & + & $22 \quad 1344-21 \quad 4054$ & 2.26 & 1.84 & 4.17 & $\mathrm{D}$ & SBa & $602-1$ & 12.70 & $2616 \pm 11$ \\
\hline $68 B$ & - & $22 \quad 1355-21 \quad 44 \quad 12$ & 1.08 & 0.70 & 13.72 & & $\mathrm{SBa}$ & $602-2$ & 14.75 & $9634 \pm 22$ \\
\hline $68 \mathrm{C}$ & + & $\begin{array}{llllll}22 & 14 & 06 & -21 & 30 & 00\end{array}$ & 2.08 & 0.70 & 12.04 & & Irr & $602-3$ & 14.70 & $2571 \pm 11$ \\
\hline $69 \mathrm{~A}$ & + & $\begin{array}{llllll}22 & 14 & 47 & -47 & 09 & 28\end{array}$ & 0.73 & 0.16 & 0.34 & $\mathbf{L}$ & $\mathrm{Sa}$ & & & \\
\hline $69 B$ & + & $\begin{array}{llllll}22 & 14 & 48 & -47 & 09 & 25\end{array}$ & 0.63 & 0.13 & 0.27 & & $\operatorname{Pec}\}$ & $289-14\}$ & 16.28 & \\
\hline $69 \mathrm{C}$ & + & $22 \quad 1448-470930$ & 0.30 & 0.10 & 0.57 & & S0-E & & & \\
\hline $70 \mathrm{~A}$ & + & $2233 \quad 50-12 \quad 4936$ & 1.57 & 0.67 & 4.33 & $\mathrm{~T}$ & $\mathrm{Sa}$ & $-2-57-22$ & 14.38 & $7504 \pm 73$ \\
\hline $70 \mathrm{~B}$ & + & $\begin{array}{llllll}22 & 34 & 07 & -12 & 48 & 20\end{array}$ & 1.12 & 0.39 & 2.18 & & S0a & $-2-57-23$ & 14.35 & $7248 \pm 11$ \\
\hline $70 \mathrm{C}$ & - & $22 \quad 34 \quad 15-124918$ & 1.01 & 0.56 & 6.09 & & $\mathrm{Sc}$ & $-2-57-24$ & & $7159 \pm 60$ \\
\hline $71 \mathrm{~A}$ & + & $\begin{array}{llllll}23 & 11 & 37 & -03 & 03 & 08\end{array}$ & 0.90 & 0.56 & 4.16 & $\mathbf{L}$ & S0a & $-1-59-4$ & & $3475 \pm 60$ \\
\hline $71 \mathrm{~B}$ & + & $231148-030000$ & 1.23 & 0.56 & 1.99 & & S0a & $-1-59-5$ & 13.85 & $3555 \pm 17$ \\
\hline $71 \mathrm{C}$ & + & $231152-025817$ & 0.78 & 0.50 & 6.12 & & Ir & $-1-59-6$ & 14.54 & $3521 \pm 47$ \\
\hline $72 \mathrm{~A}$ & + & $232101-191706$ & 1.63 & 1.40 & 12.86 & $\mathrm{~T}$ & $\mathrm{Sc}$ & $605-4$ & 14.71 & $7677 \pm 60$ \\
\hline $72 \mathrm{~B}$ & + & $232154-192006$ & 1.36 & 1.31 & 14.02 & & SOp & $605-5$ & 14.41 & $7632 \pm 71$ \\
\hline $72 \mathrm{C}$ & - & 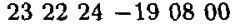 & 0.82 & 0.35 & 21.60 & & $\mathrm{Sc}$ & $605-6$ & 15.94 & \\
\hline
\end{tabular}




\begin{tabular}{|c|c|c|c|c|c|c|c|c|c|c|}
\hline$\frac{T S}{1}$ & $\frac{\text { Is. }}{2}$ & $\frac{\text { R.A.(1950) Dec. }}{3}$ & $\frac{a}{4}$ & $\frac{b}{5}$ & $\frac{X_{i j}}{6}$ & $\frac{C .}{7}$ & $\frac{\text { Type }}{8}$ & $\frac{\text { Alias. }}{9}$ & $\begin{array}{l}\overline{B_{t}} \\
10\end{array}$ & $\frac{v \pm \sigma}{11}$ \\
\hline $\begin{array}{l}73 \mathrm{~A} \\
73 \mathrm{~B} \\
73 \mathrm{C}\end{array}$ & $\begin{array}{l}+ \\
+ \\
+\end{array}$ & $\begin{array}{llllll}23 & 22 & 31 & -58 & 03 & 54 \\
23 & 22 & 47 & -58 & 09 & 42 \\
23 & 23 & 57 & -58 & 04 & 48\end{array}$ & $\begin{array}{l}1.07 \\
1.36 \\
1.40\end{array}$ & $\begin{array}{l}0.96 \\
0.61 \\
0.27\end{array}$ & $\begin{array}{c}6.17 \\
10.46 \\
11.40\end{array}$ & $\mathrm{~T}$ & $\begin{array}{l}\text { Sb } \\
\text { Sa } \\
\text { SBd }\end{array}$ & $\begin{array}{l}148-10 \\
148-11 \\
148-12\end{array}$ & $\begin{array}{l}13.44 \\
14.45 \\
14.67\end{array}$ & $3138 \pm 43$ \\
\hline $\begin{array}{l}74 \mathrm{~A} \\
74 \mathrm{~B} \\
74 \mathrm{C}\end{array}$ & $\begin{array}{l}+ \\
+ \\
-\end{array}$ & $\begin{array}{llllll}23 & 35 & 03 & -47 & 46 & 54 \\
23 & 35 & 08 & -48 & 00 & 12 \\
23 & 36 & 46 & -48 & 03 & 00\end{array}$ & $\begin{array}{l}2.44 \\
5.34 \\
1.40\end{array}$ & $\begin{array}{l}1.23 \\
0.52 \\
0.96\end{array}$ & $\begin{array}{l}13.32 \\
16.66 \\
23.58\end{array}$ & $\mathrm{~T}$ & $\begin{array}{l}\text { S0 } \\
\text { Sc } \\
\text { SBb }\end{array}$ & $\begin{array}{l}240-10 \\
240-11 \\
240-13\end{array}$ & $\begin{array}{l}12.59 \\
13.19 \\
14.00\end{array}$ & $\begin{array}{r}3189 \pm 31 \\
2843 \pm 6 \\
3246 \pm 31\end{array}$ \\
\hline $\begin{array}{l}75 \mathrm{~A} \\
75 \mathrm{~B} \\
75 \mathrm{C}\end{array}$ & $\begin{array}{l}+ \\
+ \\
+\end{array}$ & $\begin{array}{llllll}23 & 35 & 11 & -38 & 32 & 58 \\
23 & 35 & 12 & -38 & 31 & 23 \\
23 & 35 & 25 & -38 & 33 & 59\end{array}$ & $\begin{array}{l}0.90 \\
0.82 \\
0.73\end{array}$ & $\begin{array}{l}0.27 \\
0.17 \\
0.10\end{array}$ & $\begin{array}{l}1.59 \\
3.62 \\
2.92\end{array}$ & $\mathbf{T}$ & $\begin{array}{l}\text { Sbc } \\
\text { Sbc } \\
\text { Sc }\end{array}$ & $347-32$ & 15.88 & \\
\hline $\begin{array}{l}76 \mathrm{~A} \\
76 \mathrm{~B} \\
76 \mathrm{C}\end{array}$ & $\begin{array}{l}+ \\
+ \\
+\end{array}$ & $\begin{array}{lllllll}23 & 50 & 43 & -41 & 04 & 57 \\
23 & 50 & 47 & -41 & 05 & 07 \\
23 & 50 & 50 & -41 & 05 & 40\end{array}$ & $\begin{array}{l}1.08 \\
1.08 \\
0.54\end{array}$ & $\begin{array}{l}0.10 \\
0.44 \\
0.17\end{array}$ & $\begin{array}{l}0.77 \\
0.74 \\
1.49\end{array}$ & $\mathrm{~L}$ & $\begin{array}{l}\text { Scd } \\
\text { Sp\} } \\
\text { S0 }\end{array}$ & $293-8\}$ & $15.09\}$ & $9057 \pm 86$ \\
\hline
\end{tabular}

